\title{
Microstructure Evolution of a Multifunctional Titanium Alloy
}

\author{
Yu Xing Tian ${ }^{1,2} \cdot$ Yu Lin Hao ${ }^{1}$
}

Published online: 23 March 2016

(c) ASM International 2016

\begin{abstract}
To optimize both mechanical and functional properties of multifunctional titanium alloys via grain refinement, an example of such alloys termed as Ti2448 is adopted to investigate its microstructure evolution and strain rate sensitivity by compression in the single $\beta$-phase field. The results show that flow stress and strain rate follow a bilinear relation, which is in sharp contrast with other metallic materials exhibiting a monotonic linearity. Below the critical strain of $1 \mathrm{~s}^{-1}$, the alloy has a normal strain rate sensitivity factor of 0.265 . Above the critical value, its hardening rate is ultra-low with a factor of 0.03. Inspite of ultra-low hardening, the alloy is plastic stable under the tested conditions. With the aid of electron back-scattering diffraction and transmission electron microscopy analyses, microstructure evolution via several mechanisms such as dynamic recovery and recrystallization is evaluated by quantitative measurements of grain misorientation and its distribution, sub-grain formation, and localized grain refinement. These results are helpful to obtain the homogenous ultrafine-grained alloy by multi-step thermomechanical processing.
\end{abstract}

Keywords Biomedical titanium alloy $\cdot$ Microstructure evolution - Strain rate - Grain refinement - Dynamic recrystallization

Yu Lin Hao

ylhao@imr.ac.cn

1 Shenyang National Laboratory for Materials Science, Institute of Metal Research, Chinese Academy of Sciences, 72 Wenhua Road, Shenyang 110016, China

2 Chinalco Research Institute of Science and Technology, Southern District of Future Science and Technology Park, Beijing 102209, China

\section{Introduction}

The recently developed multifunctional $\beta$-type titanium alloys exhibit good functional properties of shape memory, superelasticity, zero and/or negative thermal expansion, and low Poisson's ratio, and excellent mechanical properties being flexible (low elastic modulus), strong (high strength-to-modulus ratio), and ductile [1-6]. The former is crucial for advanced functional applications and the latter is excellent for orthopedic applications to alleviate stress shielding effect by enhancement of stress transfer between implants and their surrounding bone tissues. Furthermore, this combination of properties has enormous potential for the integrated functional and structural applications such as a system for dynamic stabilization of the spine to recover its physiological function.

As a hard tissue replacing material, stronger is desirable to ensure longer service in human body. This can be achieved easily by the precipitation of the $\alpha$-phase via the aging treatment in the $(\alpha+\beta)$ phase field. However, this leads to a decrease in ductility and an increase in modulus, which is contrary to the original intention to improve strength and remain low modulus. Most important, the combined functionalities such as superelasticity and shape memory are damaged by the $\alpha$-phase precipitation.

To optimize the above mechanical and functional properties, the grain refinement would be an ideal way because of its strengthening contribution with little effect on the modulus and its modification on the martensitic transformation (MT) [7, 8]. As to the metallic materials for both industrial and biomedical applications, the microstructure is generally refined by the thermo-mechanical processing (TMP) [9, 10]. This is achieved by two mechanisms, dynamic recrystallization (DRX) and dynamic recovery (DRV). The previous results have shown 
clearly that, for the materials with low-to-medium stacking-fault energy, the discontinuous DRX (DDRX) contributes greatly to the grain refinement $[11,12]$. For the materials with high stacking-fault energy such as the $\beta$ type multifunctional titanium alloys, the microstructure evolution is dominated by DRV and continuous DRX (CDRX) [13, 14], leading to the development of abundant sub-grains. However, the DDRX refinement has been reported in several $\beta$-type titanium alloys under the condition of high strain rate [15-17]. Therefore, not only primary DRV but also DRX mechanisms of the $\beta$-type titanium alloys can be activated via the optimization of the TMP and their microstructure refinement is expected with aid of the above multiple mechanisms during hot forging and rolling.

Ti2448 (abbreviated from its weight percent composition Ti-24Nb-4Zr-8Sn) is a typical example of the multifunction titanium alloys with high strength and low elastic modulus [4]. The alloy is biocompatible because of containing only the non-toxic and non-allergic elements. Typical implants made of the alloy have been finished clinical trials in several qualified hospitals and applied for product licenses to FDA of China. The recent investigations have shown that the multiple warm-processed alloy exhibits much better balanced mechanical and functional properties due to the fabrication of a homogenous ultrafinegrained microstructure [18, 19]. In this study, the microstructure evolution of Ti2448 alloy was investigated during TMP by varying both strain rate and temperature, in hoping of optimizing the microstructure and, correspondingly, the biomechanical properties of the alloy.

\section{Experimental}

An ingot of Ti2448 alloy with a diameter of $380 \mathrm{~mm}$ was fabricated by vacuum arc melting using a Ti-Sn master alloy and pure $\mathrm{Ti}, \mathrm{Nb}$, and $\mathrm{Zr}$ as raw materials. The composition determined by chemical and gas analyses is listed in Table 1. The ingot was forged and then rolled at $850{ }^{\circ} \mathrm{C}$ to slab with a thickness of $12.5 \mathrm{~mm}$.

Cylindrical samples with a diameter of $8 \mathrm{~mm}$ and a length of $12 \mathrm{~mm}$ were cut from the hot-rolled slab and compressed uniaxially using Gleeble 3800 isothermal thermal simulator. The compression was conducted at strain rates ranged between 0.001 and $70 \mathrm{~s}^{-1}$ and

Table 1 Chemical composition of Ti2448 alloy (weight percent)

\begin{tabular}{lllllll}
\hline $\mathrm{Ti}$ & $\mathrm{Nb}$ & $\mathrm{Zr}$ & $\mathrm{Sn}$ & $\mathrm{O}$ & $\mathrm{N}$ & $\mathrm{H}$ \\
\hline Bal. & 23.6 & 3.71 & 8.37 & 0.095 & 0.0083 & 0.0033 \\
\hline
\end{tabular}

temperatures ranged between 700 and $850{ }^{\circ} \mathrm{C}$ in the single $\beta$-phase field. Prior to the test, the samples were heated to the targeted temperatures and then held for 2 min to ensure temperature homogeneity. During the compression, the instant temperature was measured by thermocouples welded to the center area of the sample surface. Nickel powder was daubed on the two end surfaces of the sample to alleviate friction. Tantalum foil with a thickness of $0.05 \mathrm{~mm}$ was laid between the anvil and the sample to prevent felting. The height reductions are selected to be 18 , 50 , and $75 \%$, corresponding to the true strain of $0.2,0.7$, and 1.4, respectively. The compressed samples were cooled in water and then sectioned along the compression axis for microstructure examination.

LEO Supra 35 field emission SEM equipped with a fully automatic electron back-scattering diffraction (EBSD) analysis system was used for the microstructure observation. The EBSD images were acquired using step sizes of $0.8-1.4 \mu \mathrm{m}$ for the overall deformation microstructure. An area of about $580 \times 430 \mu \mathrm{m}$ in central parts of deformed samples was scanned at each strain level. HKL Channel 5 software was used to perform data acquisition and post-
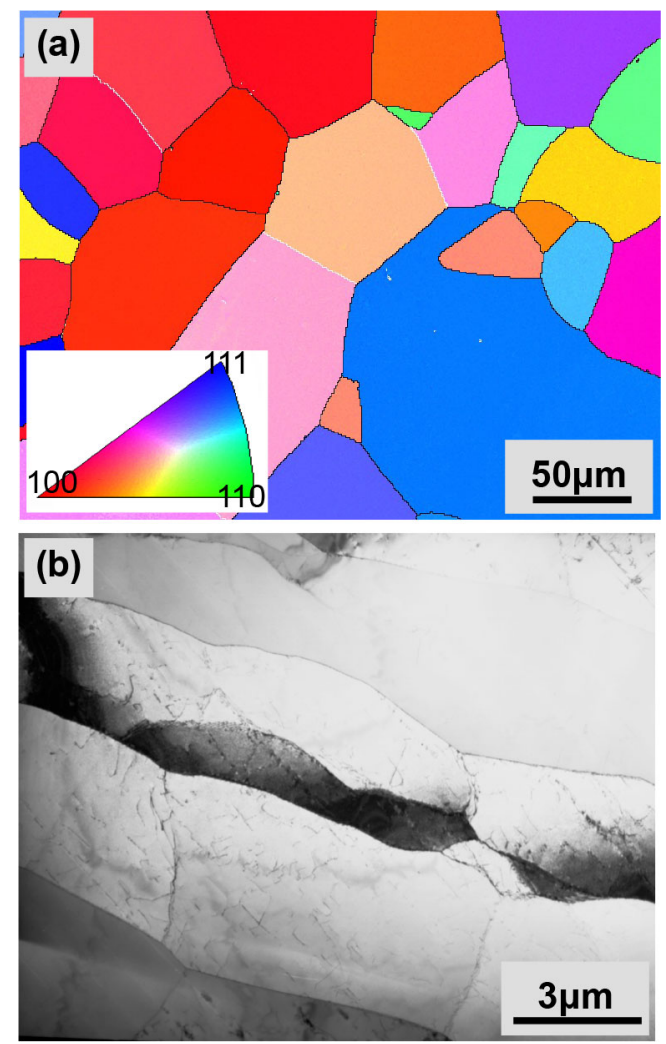

Fig. 1 EBSD (a) and TEM (b) images of the original microstructure of Ti2448 alloy. In a, crystal orientation is marked by the inset color code, and high angle $\left(>15^{\circ}\right)$ and low angle $\left(<15^{\circ}\right)$ grain boundaries are noted by black and white lines, respectively 

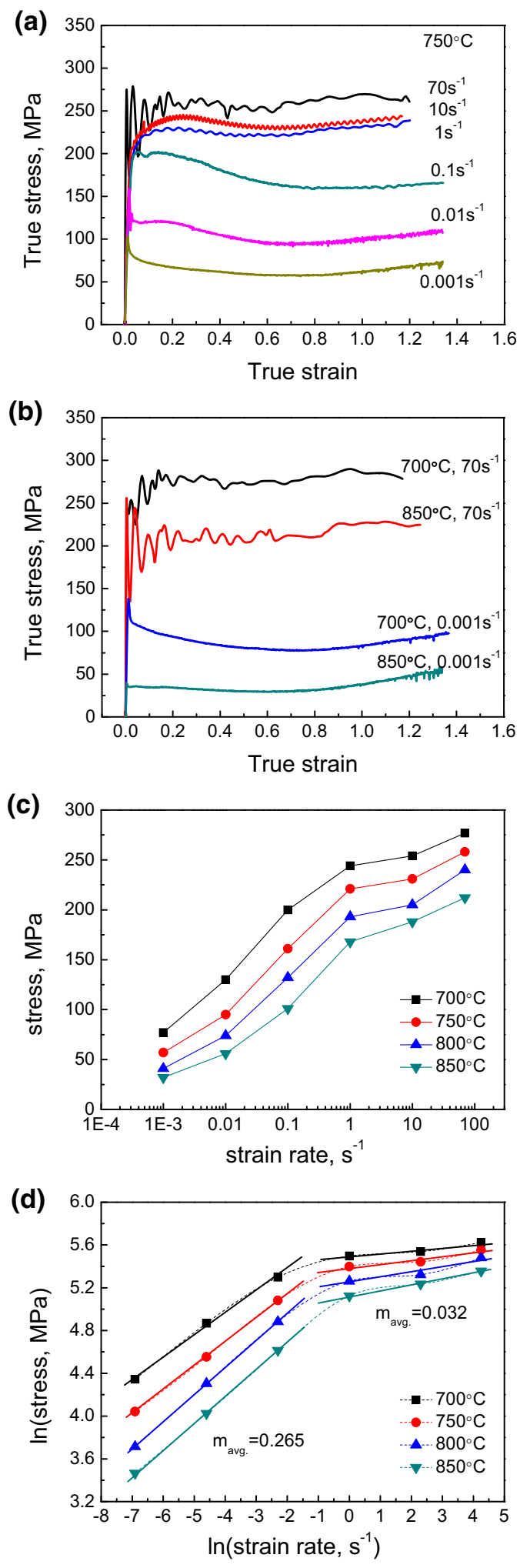

Fig. 2 True stress-strain curves $(\mathbf{a}, \mathbf{b})$ and the relationship between flow stress and strain rate $(\mathbf{c}, \mathbf{d})$

processing. Transmission electron microscopy (TEM) samples were cut from the center of deformed samples and prepared by twin-jet electro-polishing in a solution of $21 \%$
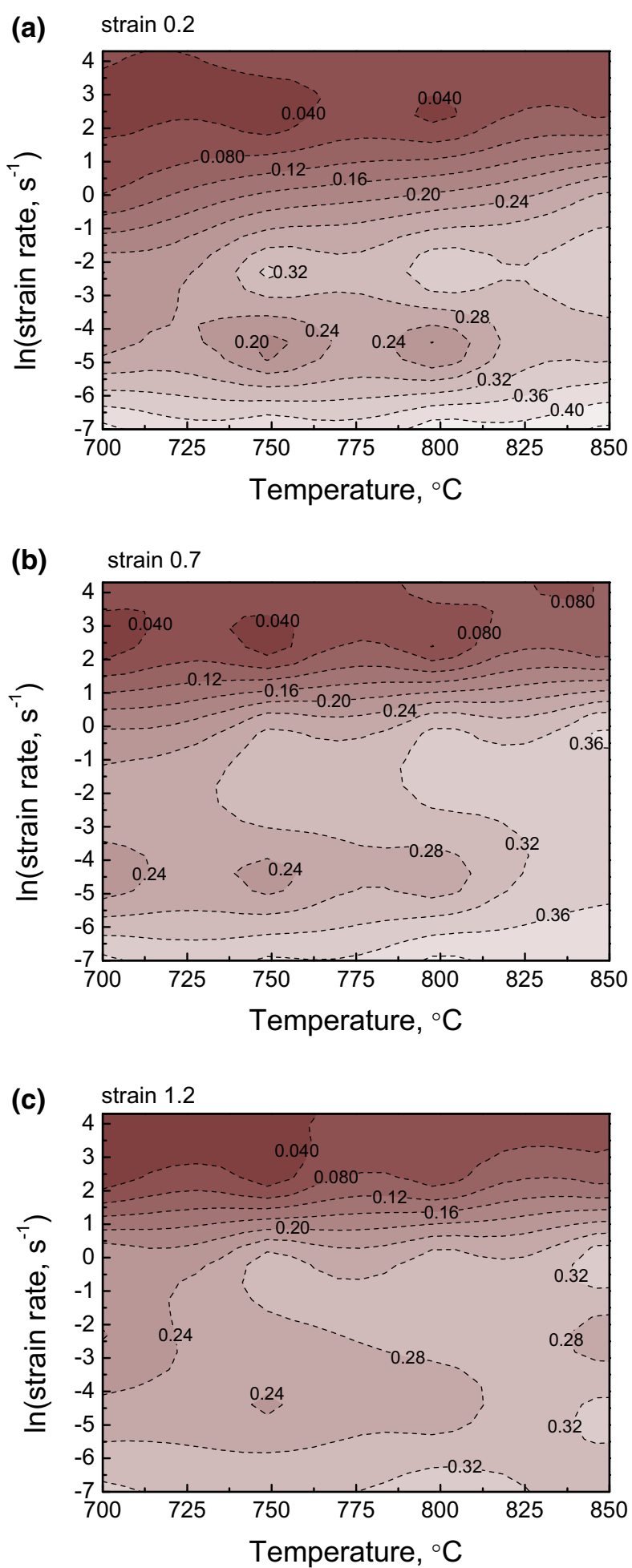

Fig. 3 The processing maps of Ti2448 alloy at the strain of 0.2 (a), $0.7(\mathbf{b})$, and $1.2(\mathbf{c})$

perchloric acid, $50 \%$ methanol, and $29 \% n$-butyl alcohol at about $-23{ }^{\circ} \mathrm{C}$ and a voltage of $15 \mathrm{~V}$. The thin foils were examined using a Tecnai G2 20 microscope operated at $100 \mathrm{kV}$. 
Fig. 4 EBSD micrographs of Ti2448 alloy compressed at $750{ }^{\circ} \mathrm{C}$ under the strain rate of $0.01 \mathrm{~s}^{-1}(\mathbf{a}-\mathbf{c})$ and $10 \mathrm{~s}^{-1}$ (d-f). The strain from the upper to below is $0.2,0.7$, and 1.4.

Elongation direction and normal direction are noted by ED and $\mathrm{ND}$, respectively
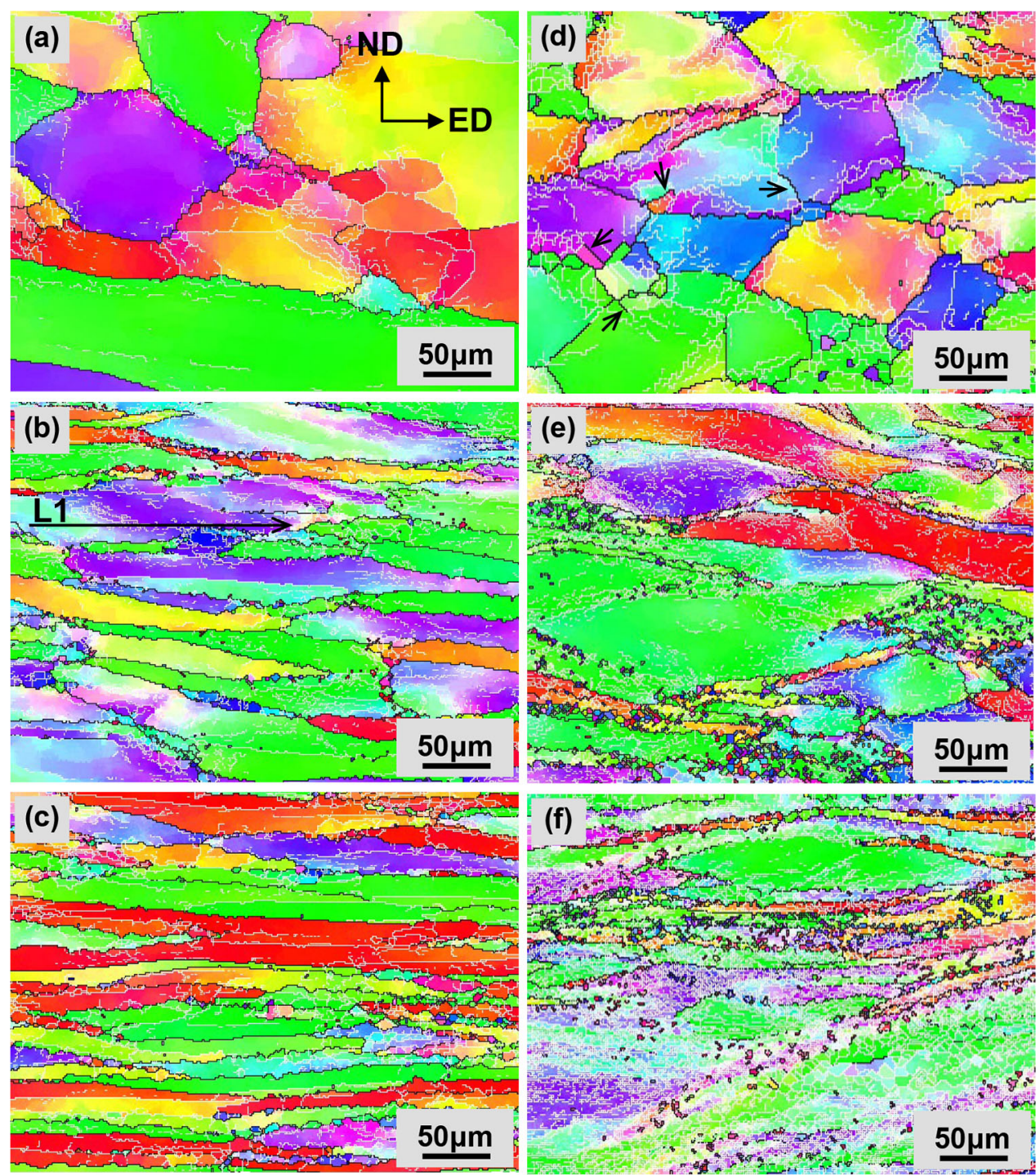

\section{Results}

\section{Original Microstructure}

EBSD image (Fig. 1a) shows that the original hot-rolled alloy has an equiaxed $\beta$-phase microstructure with an average grain size of $\sim 65 \mu \mathrm{m}$, in which most of these grain boundaries are high-angle boundaries (HABs) with a misorientation angle above $15^{\circ}$ (noted by black line) and a few are low-angle boundaries (LABs) (noted by white line). TEM observation found that these coarse grains contain many sub-grains with small misorientation angle less than $2^{\circ}$ in general (Fig. 1b), beyond the detectable limitation of the EBSD analysis. The above results are consistent with the previous report [18].

\section{Strain Rate Sensitivity}

The typical true stress-strain curves measured at $750{ }^{\circ} \mathrm{C}$ under different strain rates are shown in Fig. 2a, showing clearly a strain rate sensitivity of flow behavior. At the strain rate of $70 \mathrm{~s}^{-1}$, the flow stress rises to a peak at the beginning of loading and then reaches a multi-peak stage before the final steady state, a typical behavior of the DDRV process [20]. At the strain rates of $10 \mathrm{~s}^{-1}$ and $1 \mathrm{~s}^{-1}$, strain hardening stage exist until the steady state. As the strain rate is less than $0.1 \mathrm{~s}^{-1}$, the initial stress peak is followed by a strain softening and subsequent steady state. Such initial stress peak, which is considered generally as discontinuous yielding, is common for the $\beta$-type titanium alloys, which can be explained by a sudden generation of 
Fig. 5 EBSD micrographs of Ti2448 alloy compressed to a strain of 1.4: a $700{ }^{\circ} \mathrm{C}$,

$0.001 \mathrm{~s}^{-1} ; \mathbf{b} 850{ }^{\circ} \mathrm{C}, 0.001 \mathrm{~s}^{-1}$ c $700{ }^{\circ} \mathrm{C}, 70 \mathrm{~s}^{-1}$; d $750{ }^{\circ} \mathrm{C}$ $70 \mathrm{~s}^{-1}$
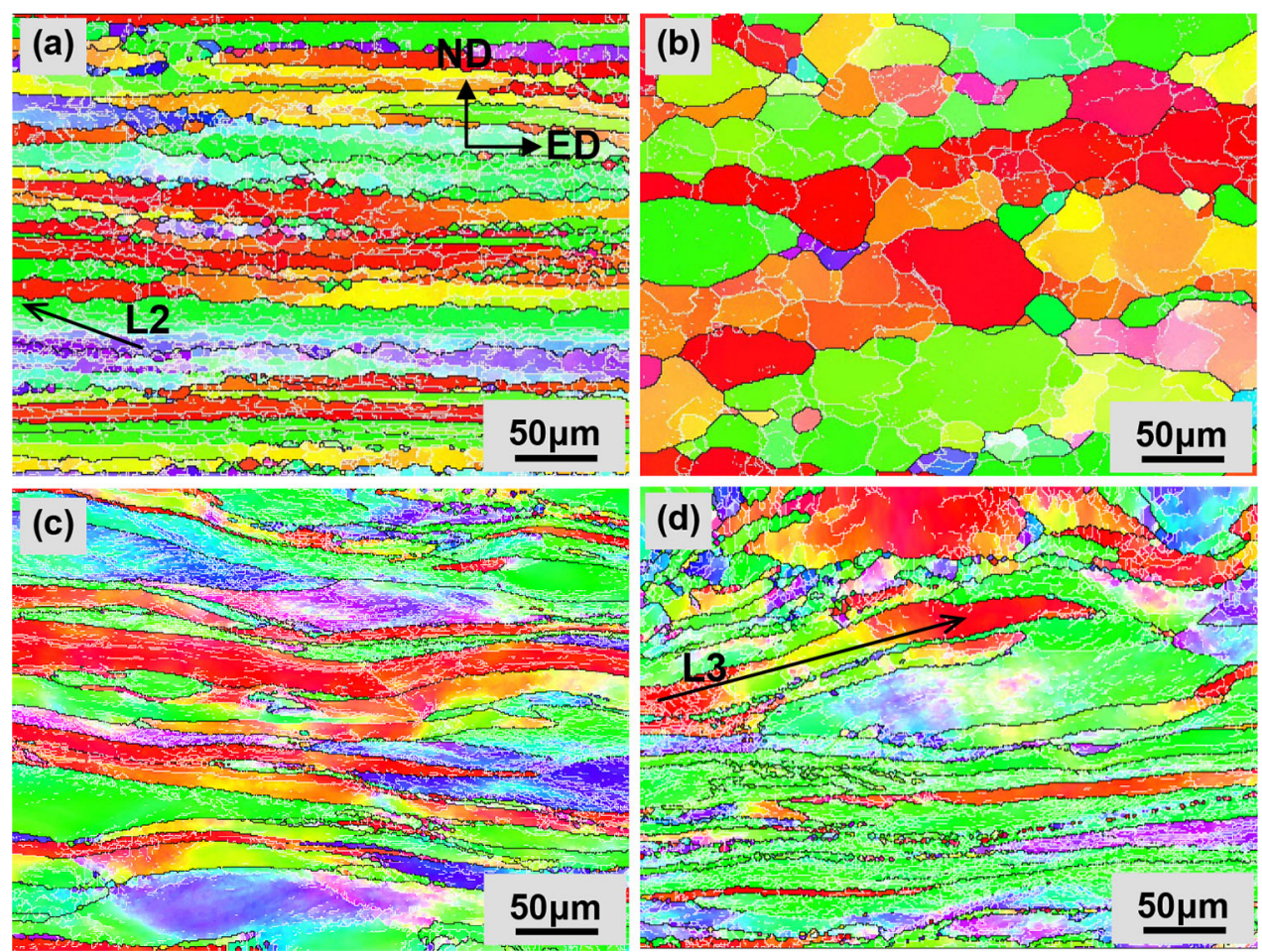

gliding dislocations [16]. Such strain rate sensitivity behavior is also observed at other temperatures, as exampled by the selected stress-strain curves shown in Fig. 2 b.

The relation between the true stress and the strain rate is shown in Fig. 2c. To estimate the strain rate sensitivity factor $m$, the above measured flow stresses and the corresponding strain rates are plotted in Fig. 2d. It is clear that there exists a critical strain rate of $\sim 1 \mathrm{~s}^{-1}$. Below the value, the factor $m$ is $\sim 0.265$, which is located at the normal range of the conventional $\beta$-type titanium alloys being 0.2-0.3 [21-23]. Above the value, however, the factor $m$ is ultra-low $\sim 0.032$. Thus, the flow stress and strain rate of the alloy follows a bilinear relation [21], which is in sharp contrast with other metallic materials obeying the monotonic linearity [22, 23]. Additionally, Fig. $2 \mathrm{~d}$ shows that the factor $m$ in both strain rate regions decrease slightly as the temperature decreased. As a result, the temperature decrease leads also to a slight decrease of the critical strain.

The above bilinear relation cannot be explained by the temperature increase caused by the compression because the data were corrected by the common method proposed by Dadras and Thomas [24]. To alleviate the effect of temperature increase, the data measured at a small strain of 0.2 were used to estimate the factor $m$ above the critical strain. The additional results confirmed also the extremely low value of $m$. Thus, the bilinear relation should be an intrinsic behavior of the alloy. In the following, we try to reveal the origin via the measured processing map, misorientation accumulation, sub-grain evolution, and localized grain refinement.

\section{Processing Maps}

To estimate the processing map of Ti2448 alloy, dynamic material model (DMM) was adopted in the study [25-27]. Since the alloy has variable factor $m$ (Fig. 2d), the Murty's criterion was applied to determine the power dissipation of the DMM and the plastic instability [28]. Thus, the power dissipated by viscoplastic heat $(G)$ is shown as Eq. (1), the power dissipation efficiency $(\eta)$ is expressed as Eq. (2) and the criterion for instable flow is deduced as Eq. (3).

$$
\begin{aligned}
& G=\int_{0}^{\dot{\varepsilon}_{\min }} \sigma \mathrm{d} \dot{\varepsilon}+\int_{\dot{\varepsilon}_{\min }}^{\dot{\varepsilon}} \sigma \mathrm{d} \dot{\varepsilon}=\left[\frac{\sigma \dot{\varepsilon}}{m+1}\right]_{\dot{\varepsilon}=\dot{\varepsilon}_{\min }}+\int_{\dot{\varepsilon}_{\min }}^{\dot{\varepsilon}} \sigma \mathrm{d} \dot{\varepsilon} \\
& \eta=2\left[1-\frac{1}{\sigma \dot{\varepsilon}}\left(\left[\frac{\sigma \dot{\varepsilon}}{m+1}\right]_{\dot{\varepsilon}=\dot{\varepsilon}_{\text {min }}}+\int_{\dot{\varepsilon}_{\min }}^{\dot{\varepsilon}} \sigma \mathrm{d} \dot{\varepsilon}\right)\right] \\
& 2 m<\eta \text { or } \eta \leq 0 .
\end{aligned}
$$

The power dissipation efficiency maps at strain of 0.2 , 0.7 , and 1.2 are shown in Fig. $3 \mathrm{a}-\mathrm{c}$. It is clear that the power dissipation exhibits a similar variation tendency as the strain increased and the $\eta$ is more sensitive to strain rate than temperature. In the high $\eta$ domain, the microstructure evolution dominates the plastic flow, while, in the low $\eta$ domain, the total power is dissipated mainly by the 
Fig. 6 Misorientation distributions of the original microstructure (a) and the deformed microstructure (b-r), in which the random distribution of cubic crystal noted by blue curve (Color figure online)

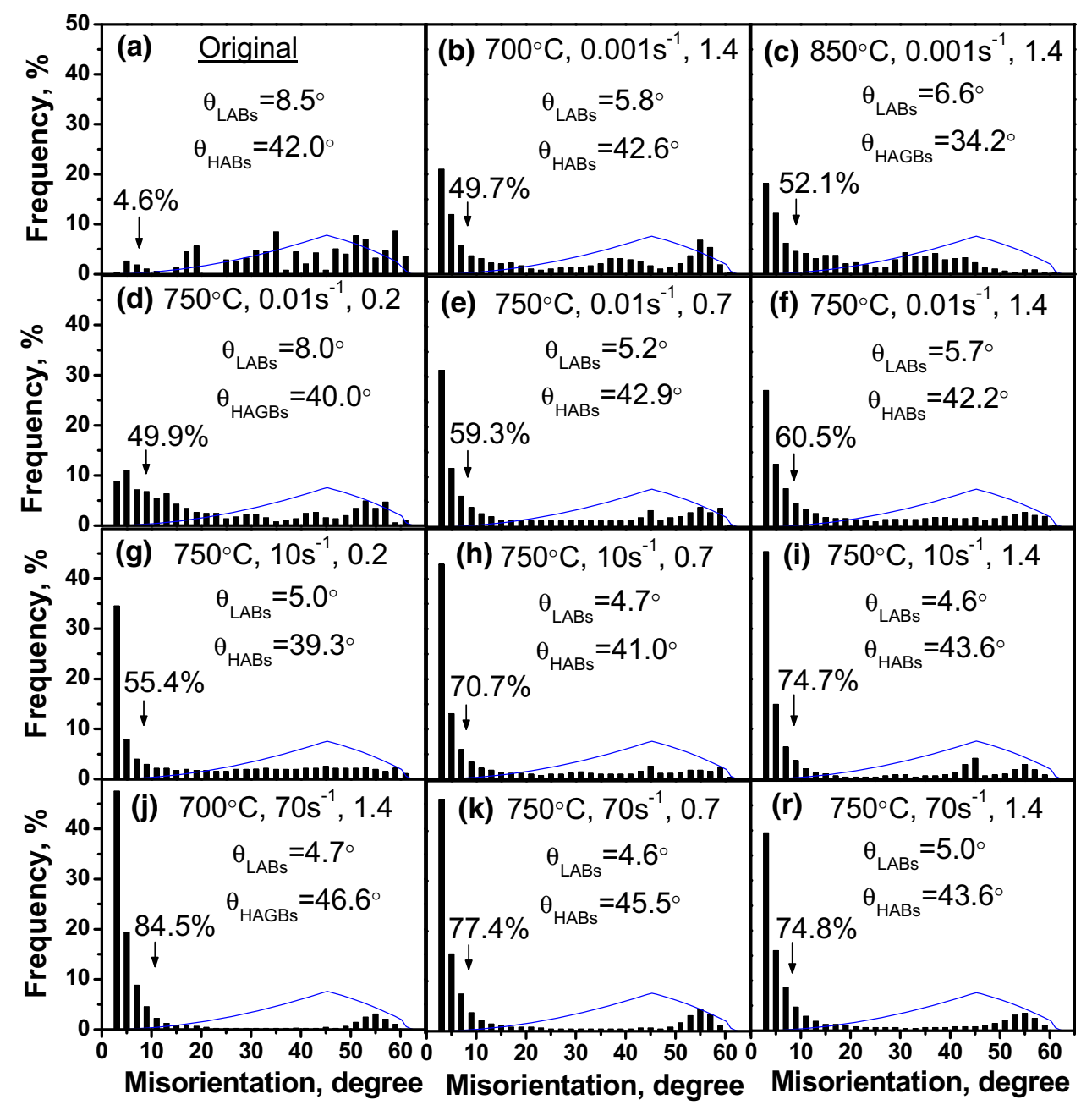

viscoplastic heat. All the $\eta$ values were kept between zero and $2 m$, suggesting stable plastic flow of Ti2448 alloy.

\section{Microstructure Evolution}

To evaluate the microstructure evolution, the samples deformed at $750{ }^{\circ} \mathrm{C}$ under the strain rate of 0.01 and $10 \mathrm{~s}^{-1}$ were selected to present EBSD images (Fig. 4). At the strain rate of $0.01 \mathrm{~s}^{-1}$, a small strain of 0.2 can induce the LABs and accommodated lattice distortion especially near to grain boundaries and triple junctions (Fig. 4a). As the strain rate increased to 0.7 (Fig. 4b), they are visible more clearly and spread from grain boundaries to the grain interiors. Several micron-scale grains appear in the original boundaries, in accordance with the CDRX process characterized by the transformation from LABs into HABs [29]. Both the strain accommodation and CDRX process are enhanced as the strain increased to 1.4 (Fig. 4c). The lattice distortion indicated by color gradient evolves into regular sub-grain boundaries, implying the primary DRV process operated at low strain rate of $0.01 \mathrm{~s}^{-1}$. Additionally, micron-scale grains with HABs can also be induced by dislocation rearrangement.

At the strain rate of $10 \mathrm{~s}^{-1}$, a small strain of 0.2 leads to more strain-induced LABs and accommodated lattice distortion than that of the low strain rate of $0.01 \mathrm{~s}^{-1}$ (Fig. 4d). The newly developed HABs at the original boundary regions (arrows noted) demonstrate that the CDRX process is operative to change the LABs into HABs $[29,30]$. As the strain rate increased to 0.7 (Fig. 4e), the original grains become pancake-shaped and contain more LABs and lattice distortion, and their boundaries are decorated by a few grain inter-layers. At 1.4 strain (Fig. 4f), micron-scale grains congregate at the triple junctions, suggesting the concentrated strain promotes the formation of LABs and accelerates the transformation from LABs into HABs [31, 32]. Thus, the DRX process including CDRX and DDRX is activated at high strain rate of $10 \mathrm{~s}^{-1}$.

The other compressed samples under different temperatures and strain rates were also observed by EBSD and 

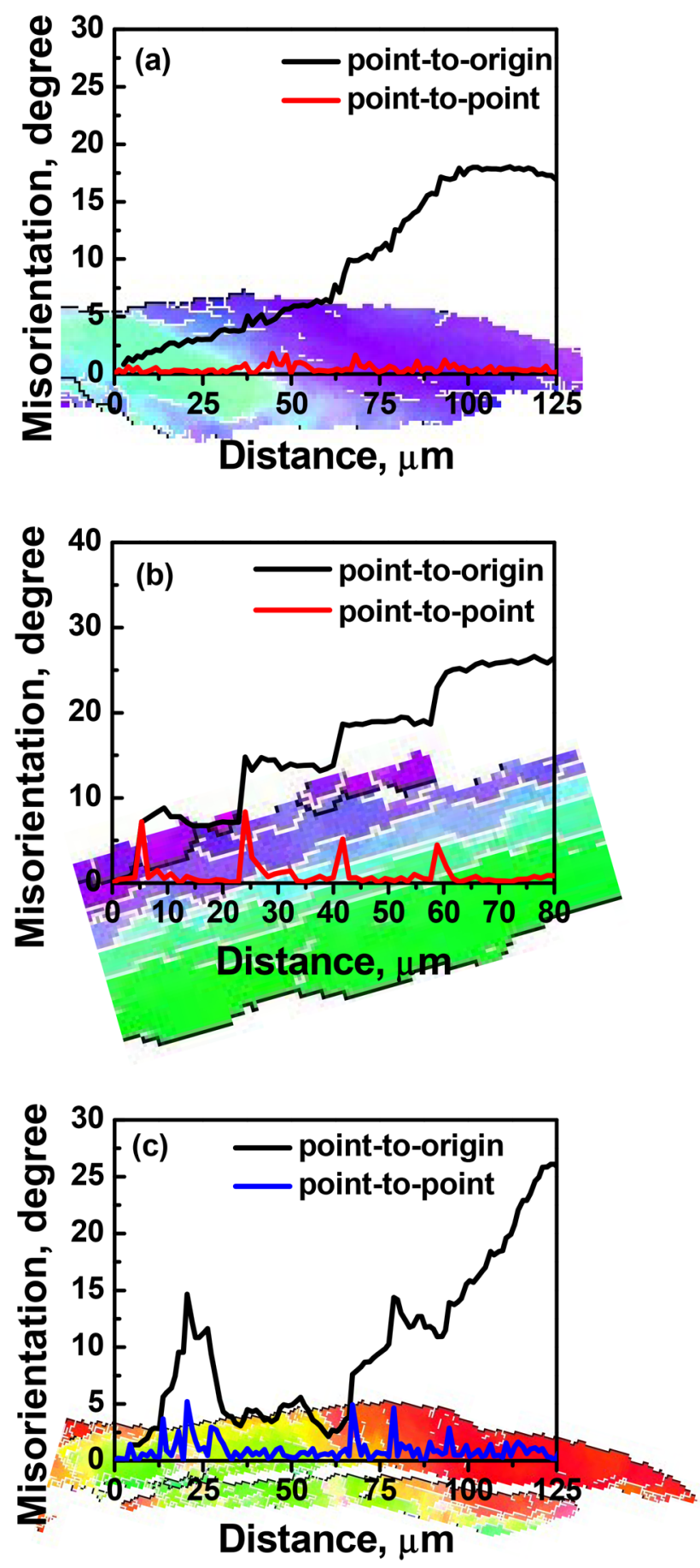

Fig. 7 Three typical misorientation accumulation patterns of continuous accumulation (a), orientation jump (b), and multi-peak orientation distribution (c), corresponding to L1 in Fig. 4b, L2 in Fig. 5a, and L3 in Fig. 5d, respectively

several examples with the strain rate of 1.4 are given in Fig. 5. The results showed clearly that the DRV is more significant at higher temperature and lower strain rate. On the contrary, the DRX is stronger.

\section{Misorientation Distributions}

To trace the microstructure evolution quantitatively, the crystal misorientations of the compressed samples were measured and the results are presented in Fig. 6. From these data, the fraction of LABs $\left(\mathrm{F}_{\mathrm{LABs}}\right)$ and the averaged misorientation angles of LABs $\left(\theta_{\mathrm{LABs}}\right)$ and HABs $\left(\theta_{\mathrm{HABs}}\right)$ were estimated (Fig. 6).

The original alloy follows roughly a random distribution of cubic crystal with small $\mathrm{F}_{\mathrm{LABs}}$ of $4.6 \%$ and $\theta_{\mathrm{LABs}}$ of $8.5^{\circ}$ (Fig. 6a). After the hot compression, the crystal misorientations become nearly unimodal distribution (Fig. 6b-r). The microstructure deformed at high strain rate of $1-70 \mathrm{~s}^{-1}$ has more $F_{\mathrm{LABs}}$ and $\theta_{\mathrm{HABs}}$ but fewer $\theta_{\mathrm{LABs}}$ than that of low strain rate of $0.001-0.1 \mathrm{~s}^{-1}$. The increased sub-grain and transformation from LABs into HABs are in agreement with the enhanced CDRX and DDRX process. Additionally, the HABs tend to be distributed randomly (as the blue lines indicated).

\section{Strain-Induced Sub-Micron Crystallites}

Strain-induced sub-micron crystallites and the localized grain refinement are two important features of the deformed microstructures because both are associated with the substructure development [33-36]. Three typical orientation accumulations, which were detected along L1, L2, and L3 directions in Figs. 4 and 5, are shown in Fig. 7. The first is a continuous orientation accumulation (Fig. 7a) that the cumulative orientation displayed by point-to-origin profile increases continuously up to about $16^{\circ}$, indicating a long-range continuous lattice distortion but the step is small, less than $2^{\circ}$ in general. The second is orientation jump (Fig. 7b), which is also called orientation band. It is clear that four sub-grains display four orientation jumps along $80 \mu \mathrm{m}$ distance (Fig. 7b). Moreover, LABs with small misorientation and lattice distortion were not observed in these sub-grains, which can be explained by a strong DRV process. The third is multi-peak orientation distribution (Fig. 7c). The point-to-origin profile exhibits multiple waves, which indicates that the lattice distortion is multidirectional rather than a long-range continuous. Furthermore, the point-to-point profile shows LABs with high density.

The three types of accumulation patterns were observed in all microstructures deformed at different strain rates and temperatures. Statistically, the first continuous accumulation is more prevalent at lower strain rate, the second orientation jump favors to low strain rate and large strains while the multi-peak orientation distribution tends to be stronger at higher strain rate. 


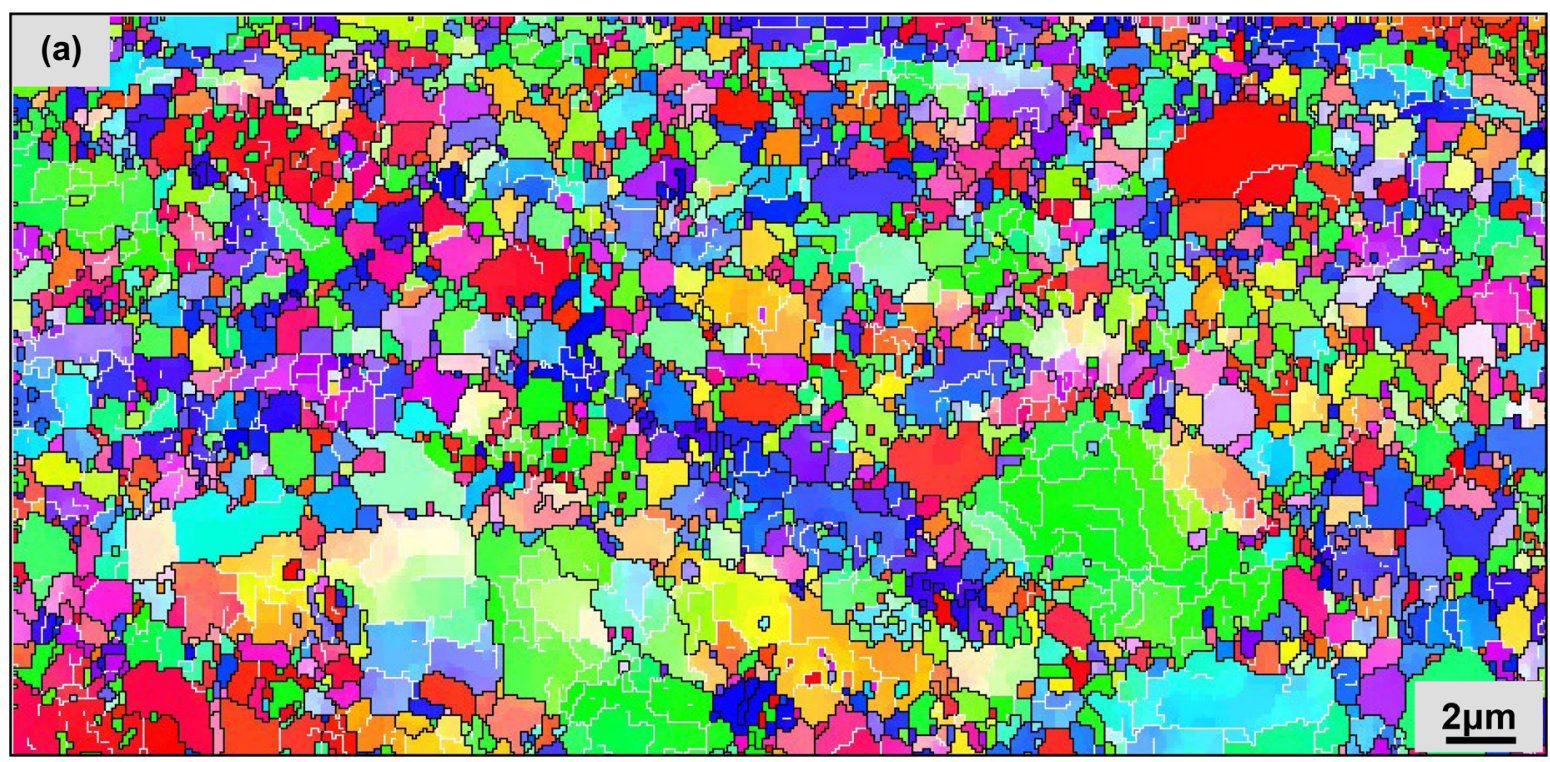

(b)

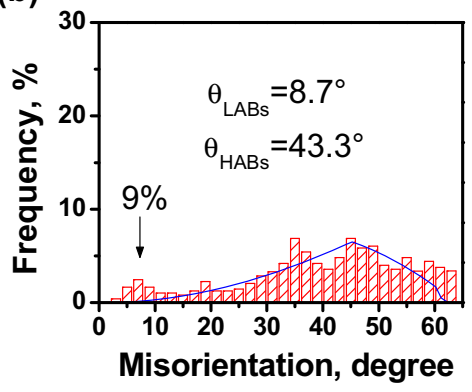

(c)

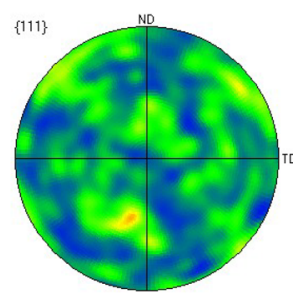

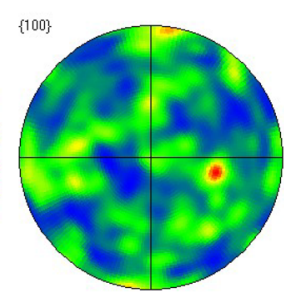

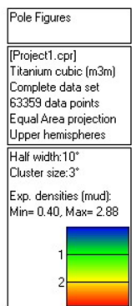

Fig. 8 Grain-refined region of the sample deformation at $850{ }^{\circ} \mathrm{C}$ under a strain rate of $70 \mathrm{~s}^{-1}$ (a) and its misorientation distributions (b) and reconstructed texture pole figures $(\mathbf{c})$

\section{Localized Grain Refinement}

The experimental results showed that the localized grain refinement is favored by both high strain rate and large deformation strain. In the localized region (Fig. 4e), the DRX grain layers align along the original boundaries and develop in the region with large strain. The localized grain refinement becomes more obvious (Fig. 4f), where the DRX layers expand into the grain interior and incline to consume the original grains. Simultaneously, the LABs with high density subdivide the elongated grains into several fragments, and then evolve into HABs by misorientation accumulation as the deformation increased.

This localized refinement is favorable to the center region of sample as deformed at $850{ }^{\circ} \mathrm{C}$ and high strain rate of $70 \mathrm{~s}^{-1}$ (Fig. 8). Figure 8a gives an example of the refined grains with HABs, containing abundant subgrains with LABs. Statistical analysis shows that these grains with size less than $1 \mu \mathrm{m}$ are about $80 \%$. Additionally, the misorientation angles of these refined grains display a random distribution of cubic crystal (Fig. 8b), being similar with that of the original coarse grains (Fig. 6a). The reconstructed pole figures also exhibit a random orientation in this localized refinement region (Fig. 8c).

\section{Discussions}

\section{The Low $m$ Value}

The strain-hardening behavior of Ti2448 alloy can be described by a bilinear relation (Fig. 2d), which is in sharp contrast with other metallic materials obeying the monotonic linearity $[22,23]$. At the low-strain rate region, the sensitivity factor $m$ is $\sim 0.265$, which is identical with other materials. However, as the strain rate over $\sim 1 \mathrm{~s}^{-1}$, it decreases sharply to an ultra-low value of $\sim 0.03$ (Fig. $2 \mathrm{~d}$ ). This novel behavior can't be explained by the transition of microstructure evolution mechanisms from DRV to DRX, although the DRX is a softening mechanism which is stronger at larger strain (Figs. 3 and 4). This is confirmed 


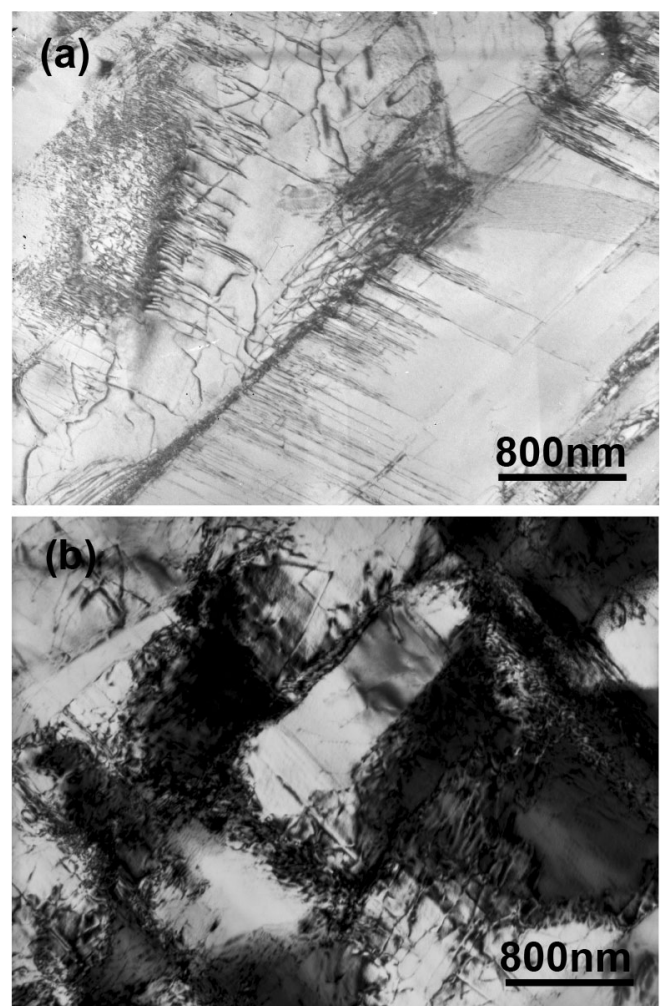

Fig. 9 Abundant dislocations (a) and cell structure (b)

further by additional data of the factor $m$ being independent of deformation strain.

The ultra-low $m$ at high temperature in single $\beta$-phase field would relate with the ultra-weak work hardening rate of the alloy at room temperature and its induced plastic instability. The previous study found that its coarse-grained microstructure can be refined easily by cold deformation and, compared with the coarse-grained alloy, the nanostructured alloy has strength higher only $\sim 5 \%[37,38]$. Since these novel behaviors are unexpected from other materials including shape memory alloys, it was termed as soft nanostructured alloy [37]. The lack of work hardening and the plastic instability at room temperature are in agreement with the alloy exhibiting an extreme high strength-to-modulus ratio reaching $\sim 2 \%$ [4], which is as high as that of these brittle amorphous materials. The in situ high-resolution TEM analyses found several novel strengthening and reversible mechanisms such as the nanoscale disturbance of BCC crystal and the homogeneously nucleated dislocation loops in advance of the formation of nano-sized martensite with equiaxed morphology [39]. These reversible mechanisms should be much sluggish because their movement can be easily detected by highresolution TEM analysis. Furthermore, the nano-sized equiaxed martensite, unlike the micron-scale internally twinned martensitic plates commonly observed in shape
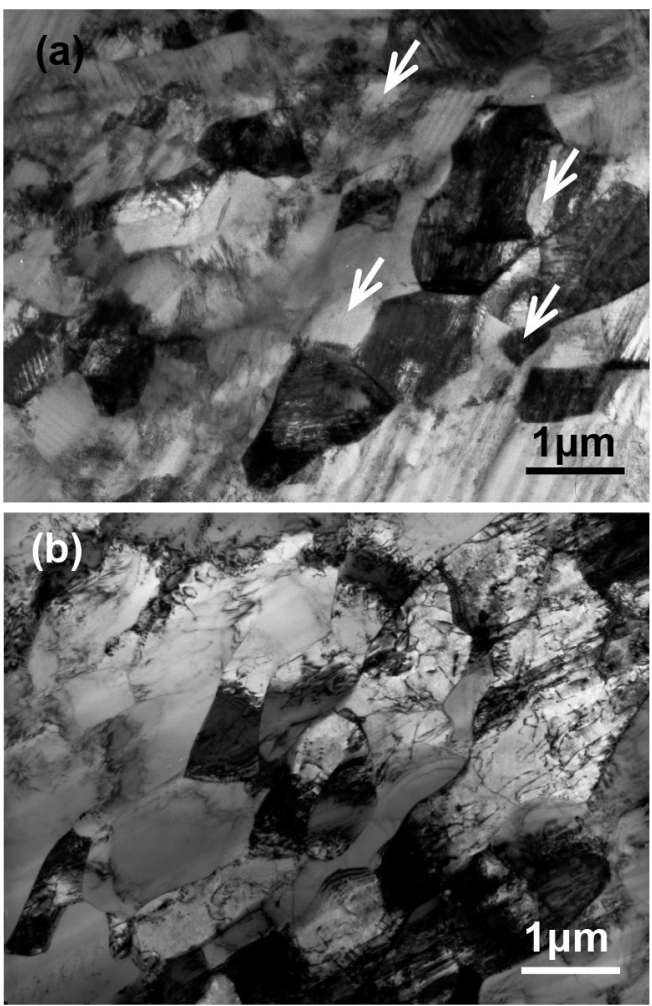

Fig. 10 TEM images of the grain-refined regions showing the DDRX grains (a) noted by white arrows and the CDRX process from LABs to HABs (b)

memory alloys, reveals the MT in the alloy is confined by unknown factors.

Under the assumption that these sluggish deformation mechanisms are operative at high temperature, the alloy should behave like other metallic materials at extreme high strain rate. This can explain the origin of its ultra-low critical strain of $\sim 1 \mathrm{~s}^{-1}$, which is lower about several orders of magnitude than other crystal materials. Since both DRV and DRX are available at high temperature, the alloy is plastic stable (Fig. 3). Thus, the microstructure evolution of the alloy can be described as the dislocation movements (Fig. 9a), cell structure formation (Fig. 9b), and then the DRX to cause the localized grain refinement at high strain rate.

\section{Localized Grain Refinement}

As the alloy compressed at the single $\beta$-phase field, the localized refinement is produced by DRX, including CDRX and DDRX. Generally, the DDRX is observed commonly in metallic materials with face-centered cubic (FCC) crystal structure, such as pure $\mathrm{Cu}$ [40], Ni-20 \% Cr alloy [12], and Nimonic 80a alloy [41], in which the grain boundary especially triple junction serve as the preferential sites for nucleation. These boundaries bugle out and prone 
to form new nucleation to satisfy the decrease in interfacial energy. For the studied alloy with BCC structure, the DDRX nucleation is enhanced by boundary bugling intensified by the concentration strain, leading to the formation of the micron-scale grains with regular boundaries and fewer dislocations (Fig. 10a). Another grain refinement mechanism is CDRX, which is considered as a continuous process started from dislocations, cell structures, and subgrains to produce the refined grains with HABs [12]. The transformation from cell boundaries to LABs, and then HABs shows a continuous dislocation accumulation process (Fig. 10b). This is similar with that of FCC metals [42, 43]. This behavior was also observed in some BCC metals under the condition of high-speed deformation [44].

Although the localized grain refinement is unusual for the studied alloy, the homogenous ultrafine-grained microstructure cannot be obtained by the single-step compression at high temperature. However, the repeated deformation such as hot forging would be possible. This was confirmed by multi-step warm deformation that the coarse grains were refined to $\sim 0.2 \mu \mathrm{m}[18,19]$. Compared with the nanostructured Ti2448 alloy, which was obtained by cold deformation [37, 38], the warm-processed alloy exhibits much better balance among strength, ductility, and superelasticity.

\section{Conclusions}

Microstructure evolution and strain rate sensitivity of Ti2448 alloy were studied by the uniaixal compression deformation at temperature of $700-850{ }^{\circ} \mathrm{C}$ and strain rate of $0.001-70 \mathrm{~s}^{-1}$. Following conclusions are drawn:

(1) The alloy has two linear strain-hardening regions separated by a critical strain of $1 \mathrm{~s}^{-1}$. The strain rate sensitivity factor is similar with other metallic materials at the low-strain region (below $1 \mathrm{~s}^{-1}$ ) but decreases about one order of magnitude to 0.03 at the high-strain region (above $1 \mathrm{~s}^{-1}$ ).

(2) The dynamic recovery (DRV) is dominant at all deformation conditions in the single $\beta$-phase field, while both high strain rate and large deformation strain favor to the discontinuous and continuous dynamic recrystallization (DDRX and CDRX).

(3) Both microstructure characterization and processing map show that the alloy is plastic stable at high temperature.

Acknowledgments This work was supported by the NBRP of China (2012CB933901, 2012CB619103), the NHTP of China (2015AA033702), the NSF of China $(51271180,51571190)$.

\section{References}

1. Niinomi M (1998) Mechanical properties of biomedical titanium alloys. Mater Sci Eng A 243(1):231-236

2. Saito T, Furuta T, Hwang JH, Kuramoto S, Nishino K, Suzuki N, Chen R, Yamada A, Ito K, Seno Y, Nonaka T, Ikehata $H$, Nagasako N, Iwamoto C, Ikuhara Y, Sakuma T (2003) Multifunctional alloys obtained via a dislocation-free plastic deformation mechanism. Science 300(5618):464-467

3. Raabe D, Sander B, Friák M, Neugebauer J (2007) Theory-guided bottom-up design of $\beta$-titanium alloys as biomaterials based on first principles calculations: theory and experiments. Acta Mater 55(13):4475-4487

4. Hao YL, Li SJ, Sun SY, Zheng CY, Yang R (2007) Elastic deformation behavior of $\mathrm{Ti}-24 \mathrm{Nb}-4 \mathrm{Zr}-7.9 \mathrm{Sn}$ for biomedical applications. Acta Biomater 3(2):277-286

5. Nakai M, Niinomi M, Zhao XF, Zhao XL (2011) Self-adjustment of Young's modulus in biomedical titanium alloys during orthopaedic operation. Mater Lett 65(4):688-690

6. Niinomi M, Nakai M, Hied J (2012) Development of new metallic alloys for biomedical applications. Acta Biomater 8(11):3888-3903

7. Hao YL, Yang R, Niinomi M, Kuroda D, Zhou YL, Fukunaga K, Suzuki A (2002) Young's modulus and mechanical properties of Ti-29Nb-13Ta-4.6Zr in relation to $\alpha^{\prime \prime}$ martensite. Metall Mater Trans A 33(10):3137-3144

8. Kent D, Wang G, Yu Z, Ma X, Dargusch M (2011) Strength enhancement of a biomedical titanium alloy through a modified accumulative roll bonding technique. J Mech Behav Biomed Mater 4(3):405-416

9. Sakai T, Jonas JJ (1984) Overview no. 35 Dynamic recrystallization: mechanical and microstructural considerations. Acta Metall 32(2):189-209

10. Weiss I, Semiatin SL (1998) Thermomechanical processing of beta titanium alloys-an overview. Mater Sci Eng, A 243(1):46-65

11. Belyakov A, Tsuzaki K, Miura H, Sakai T (2003) Effect of initial microstructures on grain refinement in a stainless steel by large strain deformation. Acta Mater 51(3):847-861

12. Dudova N, Belyakov A, Sakai T, Kaibyshev R (2010) Dynamic recrystallization mechanisms operating in a Ni-20\% $\mathrm{Cr}$ alloy under hot-to-warm working. Acta Mater 58(10):3624-3632

13. Bay B, Hansen N, Hughes DA, Kuhlmann-Wilsdorf D (1992) Evolution of fcc deformation structures in polyslip. Acta Metall Mater 40(2):205-219

14. Kumar KS, Van Swygenhoven H, Suresh S (2003) Mechanical behavior of nanocrystalline metals and alloys. Acta Mater 51(19):5743-5774

15. Philippart I, Rack HJ (1998) High temperature, high strain deformation behavior of Ti-6.8 Mo-4.5 Fe-1.5 Al. Mater Sci Eng, A 254(1):253-267

16. Balasubrahmanyam VV, Prasad YVRK (2002) Deformation behavior of beta titanium alloy Ti-10V-4.5Fe-1.5Al in hot upset forging. Mater Sci Eng A 336(1):150-158

17. Mironov S, Sato YS, Kokawa H (2010) Microstructural evolution during friction stir welding of Ti-15V-3Cr-3Al-3Sn alloy. Mater Sci Eng, A 527(29):7498-7504

18. Hao YL, Zhang ZB, Li SJ, Yang R (2012) Microstructure and mechanical behavior of a Ti-24Nb-4Zr-8Sn alloy processed by warm swaging and warm rolling. Acta Mater 60:2169-2177

19. Zhang ZB, Hao YL, Li SJ, Yang R (2013) Fatigue behavior of ultrafine-grained Ti-24Nb-4Zr-8Sn multifunctional biomedical titanium alloy. Mater Sci Eng, A 577:225-233

20. McQueen HJ, Jonas JJ (1984) Recent advances in hot working: fundamental dynamic softening mechanisms. J Appl Metal Work $3(3): 233-241$ 
21. Tian YX, Li SJ, Hao YL, Yang R (2012) High temperature deformation behavior and microstructure evolution mechanism transformation in Ti2448 alloy. Acta Metall. Sinica 48(7):837844

22. Lee WS, Lin CF, Chen TH, Hwang HH (2008) Effects of strain rate and temperature on mechanical behaviour of Ti-15Mo-5Zr3Al alloy. J Mech Beha Biomed Mater 1(4):336-344

23. Furuhara T, Poorganji B, Abe H, Maki T (2007) Dynamic recovery and recrystallization in titanium alloys by hot deformation. J Met 59(1):64-67

24. Dadras P, Thomas JF (1981) Characterization and modeling for forging deformation of Ti-6Ai-2Sn-4Zr-2Mo-0.1Si. Metall Trans A 12(11):1867-1876

25. Prasad YVRK, Gegel HL, Doraivelu SM, Malas JC, Morgan JT, Lark KA, Barker DR (1984) Modeling of dynamic material behavior in hot deformation: forging of Ti-6242. Metall Mater Trans A 15(10):1883-1892

26. Prasad YVRK, Seshacharyulu T (1998) Modelling of hot deformation for microstructural control. Inter Mater Rev 43(6): 243-258

27. Montheillet F, Jonas JJ, Neale KW (1996) Modeling of dynamic material behavior: a critical evaluation of the dissipator power cocontent approach. Mater Trans A 27(1):232-235

28. Murty SN, Rao BN (2000) On the flow localization concepts in the processing maps of titanium alloy Ti-24Al-20Nb. J Mater Pro Technol 104(1):103-109

29. Gourdet S, Montheillet F (2000) An experimental study of the recrystallization mechanism during hot deformation of aluminium. Mater Sci Eng, A 283(1):274-288

30. Belyakov A, Gao W, Miura H, Sakai T (1998) Strain-induced grain evolution in polycrystalline copper during warm deformation. Mater Trans A 29(12):2957-2965

31. Al-Samman T, Molodov KD, Molodov DA, Gottstein G, Suwas S (2012) Softening and dynamic recrystallization in magnesium single crystals during c-axis compression. Acta Mater 60(2):537-545

32. Kaibyshev R, Shipilova K, Musin F, Motohashi Y (2005) Continuous dynamic recrystallization in an $\mathrm{Al}-\mathrm{Li}-\mathrm{Mg}-\mathrm{Sc}$ alloy during equal-channel angular extrusion. Mater Sci Eng A 396(1): 341-351

33. Chen YJ, Li YJ, Walmsley JC, Dumoulin S, Roven HJ (2010) Deformation structures of pure titanium during shear deformation. Mater Trans A 41(4):787-794

34. Kaibyshev R, Sitdikov O, Goloborodko A, Sakai T (2003) Grain refinement in as-cast 7475 aluminum alloy under hot deformation. Mater Sci Eng, A 344(1):348-356

35. Jorge-Badiola D, Iza-Mendia A, Gutierrez I (2005) Study by EBSD of the development of the substructure in a hot deformed 304 stainless steel. Mater Sci Eng, A 394(1):445-454

36. Delannay L, Mishin OV, Jensen DJ, Van Houtte P (2001) Quantitative analysis of grain subdivision in cold rolled aluminium. Acta Mater 49(13):2441-2451

37. Hao YL, Li SJ, Sun SY, Zheng CY, Hu QM, Yang R (2005) Super-elastic titanium alloy with unstable plastic deformation. Appl Phys Lett 87(9):1906

38. Hao YL, Li SJ, Sun BB, Sui ML, Yang R (2007) Ductile titanium alloy with low Poisson's ratio. Phys Rev Lett 98(21):216405

39. Cui JP, Hao YL, Li SJ, Sui ML, Li DX, Yang R (2009) Reversible movement of homogenously nucleated dislocations in a $\beta$ titanium alloy. Phys Rev Lett 102(4):045503

40. Wusatowska-Sarnek AM, Miura H, Sakai T (2002) Nucleation and microtexture development under dynamic recrystallization of copper. Mater Sci Eng A 323(1):177-186

41. Tian B, Lind C, Schafler E, Paris O (2004) Evolution of microstructures during dynamic recrystallization and dynamic recovery in hot deformed Nimonic 80a. Mater Sci Eng A 367(1):198-204

42. Wang K, Tao NR, Liu G, Lu J, Lu K (2006) Plastic straininduced grain refinement at the nanometer scale in copper. Acta Mater 54(19):5281-5291

43. Luo ZP, Zhang HW, Hansen N, Lu K (2012) Quantification of the microstructures of high purity nickel subjected to dynamic plastic deformation. Acta Mater 60(3):1322-1333

44. Yasunaga K, Iseki M, Kiritani M (2003) Dislocation structures introduced by high-speed deformation in bcc metals. Mater Sci Eng A 350(1):76-80 